Review

\title{
Prognosis and management of recurrent and/or metastatic head and neck adenoid cystic carcinoma
}

\author{
Luigi Lorini $^{\mathrm{a}}$, Laura Ardighieri ${ }^{\mathrm{b}}$, Anna Bozzola ${ }^{\mathrm{b}}$, Chiara Romani ${ }^{\mathrm{c}}$, Eliana Bignotti ${ }^{\mathrm{c}}$, \\ Michela Buglione ${ }^{\mathrm{d}}$, Andrea Guerini ${ }^{\mathrm{d}}$, Davide Lombardi ${ }^{\mathrm{e}}$, Alberto Deganello ${ }^{\mathrm{e}}$, \\ Michele Tomasoni ${ }^{\mathrm{e}}$, Sara Anna Bonini ${ }^{\mathrm{f}}$, Sandra Sigala ${ }^{\mathrm{f}}$, Davide Farina ${ }^{\mathrm{g}}$, Marco Ravanelli ${ }^{\mathrm{g}}$, \\ Paolo Bossi ${ }^{\text {a, * }}$ \\ ${ }^{a}$ Medical Oncology Unit, Department of Medical and Surgical Specialties, Radiological Sciences and Public Health, ASST Spedali Civili of Brescia, University of Brescia, \\ Brescia, Italy \\ ${ }^{\mathrm{b}}$ Department of Pathology, ASST Spedali Civili of Brescia, Brescia, Italy \\ ${ }^{c}$ Angelo Nocivelli Institute of Molecular Medicine, University of Brescia and ASST-Spedali Civili of Brescia, 25123 Brescia, Italy \\ ${ }^{\mathrm{d}}$ Radiation Oncology Unit, Department of Medical and Surgical Specialties, Radiological Science and Public Health, ASST Spedali Civili of Brescia, University of Brescia, \\ Brescia, Italy \\ ${ }^{\mathrm{e}}$ Unit of Otorhinolaryngology-Head and Neck Surgery, ASST Spedali Civili of Brescia, Department of Medical and Surgical Specialties, Radiologic Sciences, and Public \\ Health, University of Brescia, Brescia, Italy \\ ${ }^{\mathrm{f}}$ Section of Pharmacology, Department of Molecular and Translational Medicine, University of Brescia, Brescia, Italy \\ ${ }^{\mathrm{g}}$ Department of Radiology, University of Brescia, Italy
}

\section{A R T I C L E I N F O}

\section{Keywords:}

Salivary gland carcinoma

Adenoid cystic carcinoma

Head and neck tumor

Prognosis

Recurrence and metastatic disease

Rare tumor

\begin{abstract}
A B S T R A C T
Adenoid cystic carcinoma (ACC) is a rare tumor, usually arising in the salivary gland, accounting for $1 \%$ of all head and neck cancers. ACC may have a long-term poor prognosis, as about $40 \%$ of radically treated patients will recur locoregionally and up to $60 \%$ will develop distant metastasis.

Factors influencing risk of recurrence have been well studied, but few data exist about prognostic factors in Recurrent/Metastatic (RM) setting. Moreover, treatment of RM ACC is often a challenge for clinicians, in the context of a rare disease, which may have an indolent clinical behavior or less frequently a quicker growth and with a paucity of available clinical trials.

This review critically analyzes pathological and molecular prognostic factors in RM ACC and make an overview on actual therapeutic choices and future direction of therapy.

Recognized prognostic factors in RM ACC are the presence and site of distant metastasis (lung vs other), the presence of nodal metastasis and of extranodal extension, skull base recurrence, disease free interval, lymphovascular invasion, solid histotypes and grading of disease, and the presence of mutation of NOTCH1 family, PI3K, and TP53.

Due to disappointing results with chemotherapy, new approaches are under study, also on the basis of biomolecular research. Ongoing clinical trials are evaluating treatment targeting MYB and NOTCH1 alterations, immunotherapy or combination of targeted treatments and immune checkpoint inhibitors.
\end{abstract}

\section{Introduction}

Adenoid cystic carcinoma (ACC) is a rare cancer, mainly originating from salivary glands; it accounts for $1 \%$ of all head and neck cancers, $20 \%$ of all malignant salivary gland tumors and $58 \%$ of minor salivary gland tumors [1].

The reported incidence of ACC is 4.5 cases/100,000 individuals, with a slight female predominance (60\%), and a median age at diagnosis of 57 years [2].

ACC may have a long-term poor prognosis, which varies depending on the retrospective studies considered. A large-scale European study showed a 10-year survival of 65\% [3], while according to another study, 5 -, 10 - and 20 -year survival rates were $68 \%, 52 \%$ and $28 \%$, respectively [4]. A large prospective French trial showed 5- and 10-year survival of

\footnotetext{
* Corresponding author at: Piazzale Spedale Civili, 1, 25123 Brescia, Italy.

E-mail address: paolo.bossi@unibs.it (P. Bossi).
} 
$85 \%$ and $67 \%$, respectively [5]. Locoregional recurrence rate is close to $40 \%$ at 5 years, while the rate of distant metastasis varies from $8 \%$ to $60 \%$ [6], with an average time to metastases of 5 years, although sometimes they can occur even 12-15 years later [7]. Metastatic pathways are mainly hematogenous, lungs ( $70 \%$ of cases), bones ( $6 \%$ of cases), liver (3\% of cases) are, indeed, the most frequently involved sites, whereas involvement in the brain or multiple sites is rare. Intracranial metastases linked to perineural diffusion along cranial nerves could be found on rare occasions [8].

Clinical behavior of recurrent/metastatic (RM) disease varies according to the site of recurrence, with generally more aggressive disease in case of locoregional recurrence than in case of distant spreading, even if exceptions to this rule may be found.

Due to the high rate of relapse and the limited treatment opportunities, many of which are not sustained by adequate clinical trials, we need to define precisely prognostic factors in the RM setting, so to give more information to clinicians in difficult therapeutic choices. In this review, we aim to analyze the prognostic relevance of several factors (clinical, pathological and molecular) in patients affected by RM ACC and to depict the possible therapeutic management.

\section{Prognostic factors}

As discussed, it is crucial to select the time and the patients to whom propose treatment for RM ACC; we should be able to depict all the clinical-pathological and molecular factors in this setting of disease as detailed as possible. This approach could ideally prompt an open discussion between patients and physicians about the meaning of any treatment opportunities and the main outcome they expect to achieve. The identification of prognostic factors is the first step in order to define a personalized treatment or an informed follow-up in each patient.

Prognostic factors in ACC could therefore be classified into factors linked to the patient, to the disease and to the treatment. Moreover, based on existing literature, they may be also divided in clinical pathological and biomolecular prognostic factors.

It should however be emphasized the fact that in such a rare disease, the evidence for prognostication is mainly derived from retrospective analysis of monoinstitutional series, therefore with intrinsic weaknesses. Moreover, one should differentiate between prognostic and predictive factors: while the former ones may be identified even with the aforementioned limits, the latter ones are lacking, even in the setting of targeted agents, as discussed later.

\section{Clinical-pathological factors}

The most important clinical-pathological factors influencing the risk of recurrence after a curative approach (tumor size, surgical margins, lymphovascular and perineural invasion, histological grade, TNM and age) $[2,9]$, have not been clearly confirmed as prognostic factors also in the RM setting.

Gao et al. confirmed the different survival rates between patients who do and do not develop distant metastasis (DM). The overall 5-, 10and 20 -year survival rates were $85.6 \%, 67.4 \%$, and $50.4 \%$, respectively, for patients without DM, and $69.1 \%, 45.7 \%$, and $14.3 \%$, respectively, for patients with DM; the median survival time after appearance of distant metastases was 36 months (range: 1-112 months) [10].

Sung et al. showed that the location of metastasis has an impact on prognosis: median survival time after appearance of DM among patients with isolated lung metastases versus those with bone metastases (with or without lung metastasis) were 54 and 21 months, respectively $(\mathrm{p}=0.04)$ [11].

Girelli et al. analyzed if the disease-free interval (DFI) from the end of treatment of primary disease and the appearance of metastases could have any influence on the prognosis. DFI $>3$ years was found to have a positive correlation with overall survival (OS). The 5 -year overall survival (OS) for patient with DFI $>3$ years was 76.5 months, compared with 47.7 for those with DFI $<3$ years [12]. A confirmation about the importance of DFI was given by Hirvonen et al., who showed that the difference in recurrence-free time interval ( $<5$ vs $>5$ years) had a significant impact on the 5-year OS and on disease-specific survival ( $\mathrm{p}<$ 0.001) [13].

In a more recent paper, a nomogram for metastatic ACC has been proposed, and it was tested and validated as prognostic tool on a population of 298 patients. Independent prognostic factors that were identified are gender, DFI and presence of lung, liver or bone metastases [14].

In a context of generally indolent disease, DFI assumes a critical role in the therapeutic choice: a shorter DFI can prompt the clinician to more aggressive treatment, rather than patients with a relapse of disease after a longer DFI.

As we lack strong prognostic factors other than what was previously identified, we tried to analyze whether prognostic factors identified in patients treated with curative intent may confirm their value when disease recurs.

How prognostic factors identified at first diagnosis could also guide the prognosis when diseases relapse is a matter of discussion. The hypothesis behind is that tumor behavior could be determined since the first appearance of the disease, not only for what concerns the risk of progression, but also for the outcome at the time of disease relapse.

At first diagnosis, radiologically or pathologically identified perineural invasion increases the risk of locoregional recurrence [15]. However, the role of the same risk factor in defining the outcome of relapsed disease has not been defined; only one study explored this parameter with respect to outcome of RM disease without finding any significant associations [7].

Lymphovascular invasion at first surgical intervention is reported in a huge range of cases, varying from $5 \%$ to $70 \%$ and it is known to carry a high risk of relapse and poor prognosis [16]. Again, limited evidence exists about its role in determining the outcome of the disease once it relapses.

The role of growth pattern and grading in guiding prognosis in locoregionally disease is well acknowledged, with high-grade ACC having a significantly lower 5-year DFS. Grading of the disease is based on histopathological components, considering tubular, cribriform and solid growth pattern, where the solid component characterizes a more aggressive disease [3,11,17-21].

Van Weert et al. confirmed the prognostic impact of grading of the disease and solid growth-pattern on 1-year OS also in the RM setting. Interestingly, in multivariate analyses, just the presence of any solid growth-pattern of disease has a negative prognostic impact on distant metastatic survival [7].

In a retrospective multicenter analysis on RM ACC patients, locoregional relapse at high risk was defined as any recurrence involving the skull base. The only factor associated with OS was skull base recurrence, therefore, identifying another possible prognostic factor in this setting [8].

A recent retrospective monocenter analysis of 125 patients who were treated for ACC, showed that 58 patients (46\%) developed DM after primary treatment. On univariate analyses factors associated with shorter OS after appearance of RM disease were lymphovascular invasion, node metastasis, extranodal extension at presentation and regional recurrence $(\mathrm{p}<0.05)$. On multivariate analyses, only regional recurrence (7.26-fold increase in risk of mortality) and extranodal extension at presentation (4.17-fold increase in risk of mortality) remained independent factors of OS after disease relapse [22].

Having considered all these factors, even with the limited amount of available data, one may underline the fact that intrinsic characteristics of primary tumor could influence the outcome also after the appearance of RM disease. This intriguing observation could suggest to carefully evaluate all the clinical factors at initial presentation of disease, and to also consider them when facing clinical choices in RM disease.

However, very limited data exist to corroborate this hypothesis, 
Table 1

Clinical-pathological factors influencing prognosis in recurrent/metastatic adenoid cystic carcinoma.

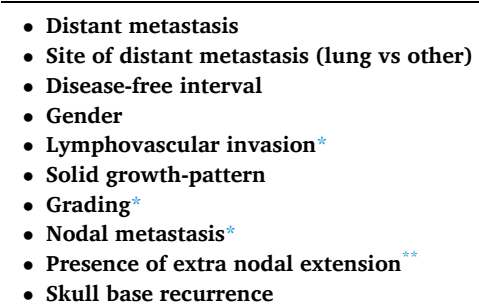

* Factors identified just in univariate analysis.

$* *$ At first diagnosis.

which, therefore, deserves further studies to be fully elucidated.

Clinical-pathological factors influencing prognosis in RM ACC are presented in Table 1.

\section{Biomolecular prognostic factors}

Biomolecular prognostic factors represent a crucial issue in ACC, both to identify patients that must be treated more intensively in case of a dismal prognosis, and to identify new therapeutic targets.

In locoregional disease, main biomolecular factors that appears to have a prognostic impact identified were NOTHC1 mutation [23]; TP53 mutation [24]; SOX2 amplification [25]; low expression of ATM [26] and EGFR mutation [27]. Despite multiple studies, the prognostic impact of fusion gene $M Y B-N F I B(\mathrm{t}(6 ; 9)(\mathrm{q} 22-23 ; \mathrm{p} 23-24))$ remains unclear $[28,29]$.

However, we have more limited information about the same molecular data when transposed to the RM setting. Moreover, data in literature show that the mutational pattern of ACC can change between primary and RM disease, emphasizing the role of biopsy on recurrence for a better selection and development of target therapy based on molecular alteration [30].

Allen et al. analyzed different genetic hallmarks between RM versus non-RM ACC. The RM ones were enriched for alterations in NOTCH (especially NOTCH1), chromatin-remodeling genes (KDMSA, MLL3 and $A R I D 1 B)$ and TERT promoter mutations.

The NOTCH family (in particular NOTCH1) alterations were found in $8 \%$ of primary ACC and $26 \%$ of the metastatic samples studied (18\% if considering only activating mutations), therefore showing a selective pressure for this alteration. Moreover, NOTCH1 mutations confirmed its negative prognostic role also when the disease relapse [31,32]. Other mutations commonly present in the RM setting and rare in the locoregional disease setting were $K D M 6 A(15.2 \%$ vs $3.4 \%$, odds ratio: 5.12 ; $\mathrm{p}$ $=0.0001$ ), MLL3/KMT2C (14.3\% vs $4.0 \%$ ), ARID1B (14.1\% vs $4.0 \%$ ), ARID1A (13.7\% vs $2.3 \%)$, BCOR (13.3\% vs $1.7 \%)$, MLL2/KMT2D (12.8\% vs $4.5 \%)$ and CREBBP ( $11.1 \%$ vs $4.5 \%)$. In particular, the KDM6A mutation showed a worse prognosis compared to patients bearing the wild-type gene.

An interesting issue emerging from this study was that in the RM setting, the alterations of $M Y B / M Y B L 1$ lost any negative prognostic impact. A mutual exclusivity of the three main genetic alterations was identified in RM ACC (NOTCH, MYB, detected in $22 \%$ and TERT, which mutated in $10 \%$ of cases), with the groups with the MYB wild-type/ NOTCH1 mutant and MYB mutant/NOTCH1 mutant carrying the worst prognosis [31].

A recent retrospective analysis on 72 cases of RM ACC showed that the most common molecular derangement was MYB alteration or rearrangement, present in $61 \%$ of cases, followed by the PI3K pathway alteration; only $5 \%$ presented with the NOTCH1 mutation. Patients with MYB alterations had better prognosis (hazard ratio: $0.23,95 \%$ CI: 0.06-0.81) compared with other subgroups, followed by patients with a disease harboring PI3K alterations and patients with NOTCH1 mutations
[30].

One should also consider the prognostic role of gene expression in ACC: according to the work of Frerich et al. the use of RNA-sequencing and gene expression could identify a subgroup of ACC patient with poor survival (median overall survival less than 30 months from diagnosis) with a gene expression signature resembling embryonic stem cells [33].

\section{When, how and why treating RM ACC}

The clinical dilemma clinicians face when considering patients with RM ACC is represented by the choice of when to start a treatment and how to select the best therapeutic weapons. To paraphrase Shakespeare, we should ask ourselves "... whether it is nobler an outrageous watchful waiting or to take arms against the disease with active therapy". In fact, the first decision to be taken is the best time to start a treatment, from one side trying to give patients a beneficial opportunity and, from the other, not harming them with too many toxicities. In this regard, it is important to consider the variables helping patients and physicians to select the most appropriate decision about any treatment choices. The Adenoid Cystic Carcinoma Research Foundation suggests a framework for treatment consideration, essentially based on the extent and location of the tumor and the tumor growth rate [34]. Basically, the site of relapse and its global burden may help to decide whether a treatment is useful within a short timeframe, due to disease that is or could early become symptomatic, as it happens for metastasis close to major bronchi or biliary tract or in bone metastasis. In addition, in case of local relapse, pain often has a high impact on the patient's quality of life, therefore prompting physicians to suggest an early treatment initiation. It is worth mentioning that surgery for locoregional recurrent disease is almost always demanding, in view of previous treatment(s), with consequent altered anatomy, and possible, multiple pathways of diffusion. Surgical treatment may have two main aims: radical excision when R0-R1 margin status may be obtained or palliation when macroscopic residual disease (R2) is foreseeable: in this last scenario improvement of obstructive symptoms and/or pain and/or delay of local progression may be pursued. Surgery with a radical intent is generally contraindicated when excision should imply an unacceptable morbidity for the patient or when dealing with a concomitant, massive burden of distant metastatic disease. On the other side, the presence of only lung metastasis, without any risks of symptoms in a short time, could suggest a watchful waiting strategy. Tumor growth rate is the other parameter to be considered. A quick tumor progression, even if in an asymptomatic patient, could represent a turning point for choosing to activate treatment in a previously indolent disease. In this regard, the calculation of the tumor volume doubling time could be an interesting tool to be developed in RM ACC, by analogy with thyroid carcinoma metastatic to the lung, where this parameter could help evaluating disease aggressiveness and need of starting systemic therapy [35].

However, we should be aware of the fact that in most cases of metastatic disease, treatment has no ambition of curing the patients and, even worse, it has no role in prolonging survival. Gao et al showed in a monoinstitutional series of metastatic ACC that the prognosis was similar between patients who received treatment for metastasis and those who did not [10]. Therefore, in many cases, we should move the focus of our therapeutic choice from the question of "how to treat" to "why should we treat" RM ACC patients.

As it is unclear whether systemic treatment or other approaches alter the natural history of RM ACC, the primary goal of any therapies should be the palliation of disease-related symptoms.

\section{Treatment}

Traditionally, ACC has been considered as an indolent disease; however, it sometimes shows a relentless behavior, which makes the identification of patients in need of a treatment or just to be followed-up closely challenging. As previously expressed, this is a clinical dilemma 


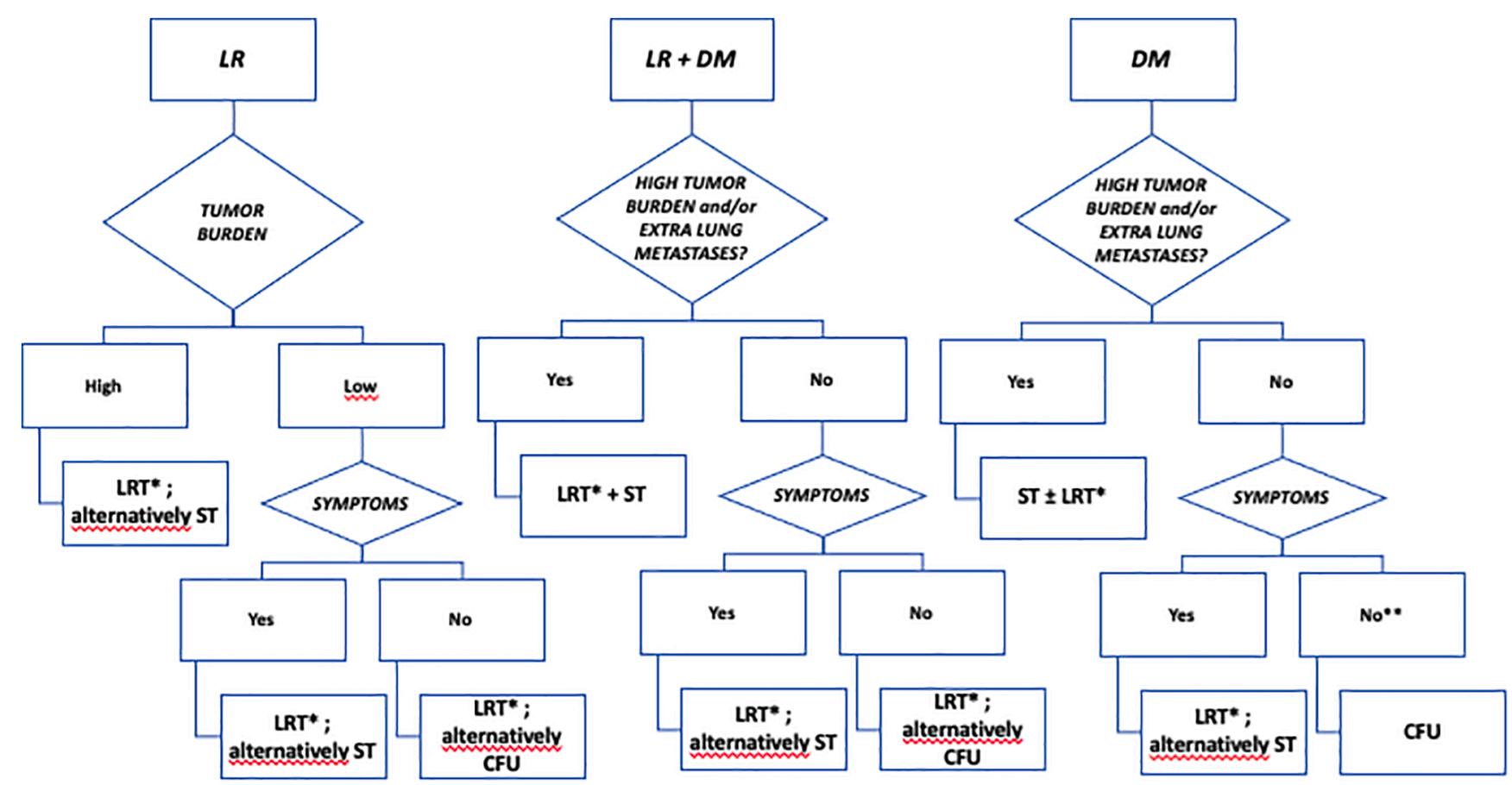

Fig. 1. Flow chart for management of RM ACC. Notes: Choice of treatment should be tailored according to previous therapies received; consider also DFI to choose the most appropriate treatment/follow up. * Surgery; SBRT; chemoembolization; radiofrequency ablation. Legend: LR (locoregional recurrence); DM (distant metastasis recurrence); SBRT (Stereotactic Body Radiation Therapy); LRT (Locoregional treatment); ST (Systemic Treatment); CFU (Close Follow Up).

the physician faces when approaching such a disease. It should be however underlined as a general principle that different types of relapse should be approached in different ways: locoregional relapse alone needs a more aggressive radiotherapeutic or surgical treatment approach than oligometastatic lung disease. Surgery or radiotherapy may be part of the treatment of locoregional relapses. Similarly, widespread metastatic ACC requires systemic treatment, while limited bone involvement would need radiation therapy alone

Literature data about treatment of RM ACC are scant and often lacking homogeneous and precise selection criteria. In some series, locoregional recurrent disease has been included in the same group of metastatic patients; moreover, progression in the last months has not been always considered as a parameter to include patients in trials with systemic therapy, therefore leading to enrolment of patients with heterogeneous prognosis. In addition, due to the rarity of the disease even more in the relapsed setting, clinical studies enrolled a limited number of patients [36]. Again, as previously expressed, the key question is whether any treatments could impact on the natural history of RM ACC. Recently, Hanna et al. showed, in a longitudinal series of 72 RM ACC, no influence on outcome of the systemic or locoregional treatments applied, as patients who received at least one systemic treatment had no improved prognosis compared with those who were only on surveillance (hazard ratio: $2.01 ; \mathrm{p}=0.35$ ) [37]. In addition, they showed that patients treated with systemic treatments according to their molecular profile did not perform better than patients treated with other systemic treatments. Hereafter, we will analyze different therapeutic approaches available and the possible role of new systemic treatments within ongoing clinical trials.

In Fig. 1 we propose a treatment flow chart for RM ACC, based on site of recurrence or metastases, tumor burden and presence of symptoms.

\section{Surgery on distant metastasis}

The role of metastasectomy remains controversial. There are limited data about surgical excision of metastases in ACC and mainly when the disease is confined to the lung. Recently, a retrospective study investigated the role of metastasectomy in patients with pulmonary disease, and showed that along a time period longer than 20 years, the two factors associated with better prognosis were a DFI $>3$ years and no residual disease after metastasectomy [12]. Neither solid data are available for other locoregional procedures in case of lung metastasis, nor other treatments have been clearly studied for metastatic ACC in other sites; indeed isolated case reports exist for localized treatment, such as embolization or radiofrequency in case of liver metastasis [38].

\section{Radiotherapy on recurrence or distant metastases}

\section{Local recurrence}

Radiotherapy is often indicated after surgery for local or nodal recurrence, especially if recurrence is multiple and multicentric, if nodal involvement with extra-capsular spread is evident, if perineural spread till skull base is present and/or if surgery is not indicated due to patient's frailty or to possible surgical morbidities. Post-operative radiotherapy reduces the rate of local recurrence in primary disease approach; even if we lack data in RM disease, its use may therefore also be suggested at recurrence $[39,40]$. Radiotherapy alone has proved to be an effective alternative when surgery is not feasible as primary treatment and has been suggested also to treat recurrent ACC with "curative" intent; retreatments are also feasible with both photons and proton beams [41].

Heavy-particle radiotherapy (eg. carbon ions, protons) can be useful, in selected cases, in reducing dose to organs-at-risk (OAR) while maintaining it to the target [42].

Its limitations are, however, similar to photon radiotherapy (OAR's dose in already treated fields) and further it is evident the difficulty to use heavy particles in adjuvant setting.

\section{Distant metastases}

As for surgery, the role of local treatments on single metastasis or oligo-metastatic disease may be discussed. However, no specific data exist on metastatic ACC. Data from randomized and non-randomized 
trials on different histologic types can be extrapolated in this setting of patients, employing different photon radiotherapy techniques to precisely deliver very high doses on the site of disease while completely sparing the nearest OAR $[43,44]$.

Data in literature pointed on head and neck cancer in general, even if not on ACC salivary gland in particular, are confirming the possible benefit in terms of disease control and the safety of this therapeutic option $[45,46]$.

Anyway, any procedure of metastasectomy or locoregional treatments should be considered only after having carefully considered the growth rate of the disease, if there are symptoms requiring palliation, what the expected results of the procedure(s) are, how residual organ function can be affected and whether there are reasonable opportunities to impact on the spread of the disease.

The objectives of any ablative local treatments should thereby be the possibility to lower the pace of disease, the delay in starting any systemic treatment and the palliation of symptoms, if necessary.

\section{Systemic therapies}

\section{Chemotherapy}

Systemic chemotherapy for RM ACC patients remains a challenge, as the slow-growing characteristics of the disease make it, on average, poorly responsive to these agents. Therefore, it is generally wellaccepted that standard chemotherapy is reserved for patients with symptomatic metastases or rapidly growing disease [47]. No randomized trial has been conducted in this setting of disease and data are extrapolated from studies, including multiple histological subtypes of salivary gland cancers. Globally, studies confirmed that a two-drug polychemotherapy with platinum plus anthracycline gives better response rate compared with monotherapy (25\% vs $15 \%$ ); a three-drug regimen (e.g. CAP scheme with cisplatin, doxorubicin and cyclophosphamide) has never proven to be more active and efficacious than a twodrug combination [48-50].

The most common response to treatment is stable disease, as it can be reasonably expected from a disease with a low tumor proliferative index. After the failure of the first-line chemotherapy, there are no viable alternatives as second-line chemotherapy [36]. Taking all this into account, chemotherapy should be reserved to symptomatic patients or in case of fast-growing disease, as studies conducted on a non-selected RM ACC population of patients were not able to identify an improvement in OS with this approach [51]. Further research is needed focused on the identification of the subgroup of patients with a more aggressive disease, who could theoretically benefit from a systemic chemotherapy.

\section{Targeted therapies}

Several trials have evaluated the use of targeted agents acting on different pathways in the carcinogenetic process of ACC or leveraging on the molecular alterations shown in preclinical studies. In this regard, we should acknowledge the low mutational burden of ACC and, till now, the lack of a targetable driver mutation, which could change the natural history of disease. Overall, targeting Epidermal Growth Factor Receptor (EGFR), cKIT, proteasome, Fibroblast Gowth Factor Receptor (FGFR) and Protein Kinease B $(A K T)$ pathway with single agents was not able to produce a dramatic response rate and survival benefits. In this regard, several drugs have been tested mainly within the framework of small phase II trials, aimed at verifying the activity of specific compounds in ACC. Cetuximab [52], imatinib [53], bortezomib [54], nelfinavir [55], dovitinib [47,56] and dasatinib [57] as single agents showed limited response, if any, and to a larger extent stabilization of disease.

Combination therapies with chemotherapy and imatinib or bortezomib have been explored, without showing synergistic effects $[54,58]$.

There is a quite strong rationale to employ agents acting on neovascularization in ACC. In fact, an association between Vascular
Endothelial Growth Factor (VEGF) expression and worse survival has been shown [59]; moreover, mutation of NOTCH1 showed to induce neoangiogenesis and high microvessel density, as mechanism of tumor growth and metastatization [60]. Several agents acting on this pathway have been tested.

Sorafenib was studied in two phase II trials in 19 and 23 RM ACC patients: overall, median progression-free survival (PFS) of 8.9 and 11.3 months and a median OS of 26.4 and 19.6 months were reported, respectively. Therapy was aggravated by grade 3 or greater side effects in $20 \%$ and $50 \%$ of patients, respectively. The response rate was similar, $15 \%$ and responders showed an enriched stromal component of PlateletDerived Growth Factor (PDGFR) $\beta[61,62]$.

Axitinib, a second-generation antiangiogenic drug, was tested on ACC patients with a response rate of $10 \%$, without significant advantage in OS compared to historical data with chemotherapy [63]. Recently, the results of the first randomized trial with an antiangiogenic drug in ACC have been reported [64]. The trial randomized patients with disease progression within the last 9 months to axitinib $10 \mathrm{mg}$ or observation, with the possibility to crossover to axitinib at progression. The 6month PFS rate was $73 \%$ in treatment vs $23 \%$ in control arm, while no difference on OS appeared, due to the crossover effect; mutation burden or any specific mutation were not correlated neither to response nor to PFS.

Lenvatinib, an oral multitarget tyrosine kinase inhibitor, with a significant inhibition of VEGFR, c-KIT, FGFR and RET has been recently tested on ACC in two phase II studies $[65,66]$. The rational of targeting these multiple pathways relies on the upregulation induced by MYB of several downstream genes, including VEGFA, FGF2 and KIT, compared to physiological glandular tissue [55]. The results of the first trial showed $75 \%$ of patients achieving disease stabilization and $15 \%$ a partial response, with a median PFS of 17.5 months; genomic profiling did not show any predictive activities [65]. Toxicity of the treatment should be considered, as $62.5 \%$ of the patients experienced at least one grade 3-4 adverse events and one out of four patients withdrew consent from the study before progression for drug-related issues. An Italian group reported the results of another phase II clinical trial with lenvatinib in the same setting of patients. A partial response was identified in $11.5 \%$ of the patients and $77 \%$ had stable disease, with a median PFS of 9 months; grade 3 adverse events were reported in approximately $50 \%$ of patients and quality of life at 6 months showed deterioration mainly in the domains of fatigue and dry mouth [66].

Overall, these observations seem to indicate a role of antiangiogenetic drugs in RM ACC in disease control, which should be carefully balanced against the toxicity of these drugs, the impact on quality of life and the limited response rate.

The results of a phase II trial with cabozantinib in 25 patients with cMET-positive RM salivary gland cancer, comprising ACC, has been recently reported, with a response rate of $6 \%$ in ACC patients, PFS of 12.6 months with severe toxicity. Because of this toxicity ( $32 \%$ of patient developed severe wound or fistula) the study has been prematurely closed [67].

The low burden of mutation in ACC, both in oncogenes and tumorsuppressor genes, underlines the fact that other mechanisms may be implicated in carcinogenesis. A few recurring mutations have been reported in genes involved in chromatin remodeling regulations, and changes in chromatin structure have been implicated in ACC [31,68]. These epigenetic changes could pave the way for treatment with drugs acting on this mechanism. The histone deacetylase (HDAC) inhibitor vorinostat has been employed as single agent in a prospective trial, showing a limited response rate $(7 \%)$ and a 6-month rate of disease stabilization of $75 \%$ [69]. However, HDAC inhibitors could exert a synergistic effect when employed in combination therapy. In this regard, use of vorinostat and cisplatin in preclinical models showed an enhanced activity, depleting cancer stem cells and reducing tumor viability [70]. In addition, the association of HDAC and immune checkpoint inhibitors is justified by the ability of the former to upregulate PD-1 expression. 
Table 2

Ongoing clinical trials open to patients with recurrent/metastatic adenoid cystic carcinoma.

\begin{tabular}{|c|c|c|c|c|c|}
\hline Study & Drug & Setting/phase study & Primary endpoint & $\begin{array}{l}\text { Estimated enrollment (number } \\
\text { of patients) }\end{array}$ & $\begin{array}{l}\text { Study completion } \\
\text { date }\end{array}$ \\
\hline NCT03287427 & $\begin{array}{l}\text { TetMYB vaccine + BGB-A317 } \\
\text { (anti-PD-1) }\end{array}$ & $\begin{array}{l}\text { Advanced/metastatic colorectal disease or } \\
\text { ACC, phase I }\end{array}$ & Safety & 32 & $30 / 12 / 22$ \\
\hline NCT03691207 & AL101 & $\begin{array}{l}\text { RM ACC with NOTCH1/2/3/4 mutation, } \\
\text { phase II }\end{array}$ & ORR, CR, PR & 87 & $01 / 06 / 21$ \\
\hline NCT03172624 & Nivolumab + Ipilimumab & RM SGC including ACC, phase II & $\begin{array}{l}\text { Best overall } \\
\text { response rate }\end{array}$ & 64 & $31 / 05 / 21$ \\
\hline NCT04209660 & Lenvatinib + pembrolizumab & RM ACC or other SGC, phase II & $\begin{array}{l}\text { Best overall } \\
\text { response rate }\end{array}$ & 64 & $31 / 12 / 22$ \\
\hline NCT03360890 & Docetaxel + pembrolizumab & $\begin{array}{l}\text { Poorly chemo-responsive thyroid and salivary } \\
\text { gland tumors, phase II }\end{array}$ & $\mathrm{RR}$ & 46 & $30 / 09 / 20$ \\
\hline NCT03990571 & Axitinib + avelumab & RM ACC, phase II & ORR & 30 & $30 / 12 / 22$ \\
\hline NCT04119453 & Rivoceranib (apatinib) & RM ACC, phase II & ORR & 72 & $01 / 02 / 24$ \\
\hline NCT04291300 & Lutetium-177-PSMA & RM ACC and SGC, phase II & Safety & 10 & $01 / 05 / 24$ \\
\hline
\end{tabular}

ACC: Adenoid cystic carcinoma; CR: Complete response; ORR: Objective response rate; PR: Partial response; RM: Recurrent/metastatic; RR: Response rate; SGC: Salivary gland cancer.

Notwithstanding these premises, in a phase II study pembrolizumab and vorinostat showed limited activity, as only one partial response out of 12-treated patients was obtained [71].

Given the relatively high frequency of the translocation MYB-NFIB in ACC, targeted drugs against pathways activated by MYB have a strong rationale. Some signs of activity have been demonstrated with $9 \%$ of partial response, $76 \%$ stable disease and a median PFS of 5.7 months, with a trend toward superior PFS without statistical significance in patients with MYB/NFIB rearrangement treated with axitinib [72]. A study is currently ongoing evaluating a potential association between immunotherapy (anti-PD-1) and MYB DNA vaccines in various diseases, including ACC (NCT03287427).

Another therapeutic target in MYB translocation-positive ACC could be the IGF2-IGF1R signaling, but the promising results obtained with the use of IGFR1 inhibitors in vitro have not been confirmed in vivo, yet [73].

RM ACC is enriched for alterations in genes of the NOTCH family and these aberrations are associated with a worse prognosis and more aggressive disease $[31,32,74,75]$. Therefore, several trials have been designed to address this pathway in ACC.

A phase I study of crenigacestat (NOTCH inhibitor) showed a manageable safety profile, pharmacodynamic effect but no objective response [76]. Another oral NOTCH inhibitor (CB-103) is under study in a phase IIa study (NCT03422679).

Brontictuzumab is a humanized monoclonal antibody directed against NOTCH-1 receptor with potential antineoplastic activity; it has been tested in a phase I study with evidence of partial response in two out of 12 RM ACC patient and disease stabilization in three out of 12 cases [77]. The ACCURACY trial is evaluating AL101 in RM NOTCHmutated ACC (NCT03691207). Recently, data of the first treatment cohort have been presented, showing diarrhea, nausea, and fatigue as main toxicities, even if of low grade; one patient died due to pneumonia, possibly treatment related. Partial response was identified in $15 \%$ of the cases [78].

ACC may be included in basket studies targeting specific mutations that may be noticed. In this regard, PARP inhibitors as talazoparib are being studied in various tumors, including ACC with BRCA mutation (NCT01989546, study completed, awaiting for results); a review on genetic hallmarks of ACC recurrence metastatic showed a $4 \%$ of germline mutation of BRCA1 or BRCA2 in ACC cases [31]. Role of PARP inhibitors in MYB alterated ACC could be reinforced by the trial of Andersson et al which showed that DNA damage sensor kinase ATR resulted overexpressed in MYB alteraed ACC and could be a potential therapeutic target. Moreover, ATR inhibition is a known mechanism to overcome PARP inhibitor resistance in other histologies [79]: ATR inhibitor in association with PARP inhibitor could be a potential therapeutic target for MYB altered RM ACC [80].

Lastly, an in vitro study hypothesized a possible role of association of
CDK inhibitor dinaciclib with chemotherapy to improve anti tumoral effect and minimize side effect [81].

\section{Immunotherapy}

Immunotherapy is becoming more and more important in the oncological panorama. Molecular and histological characteristics suggest a low immunogenicity of ACC, having a low tumoral mutational burden, low tumor-infiltrating lymphocytes, low dendritic cells and low levels of PD-1+ and CTLA4+ cells [82-85]. Preliminary data from pembrolizumab in RM ACC in the KEYNOTE028 study showed a 6month OS of $76 \%$ and a 6 -month PFS of $17 \%$. At a follow-up of 20 months, the response rate was $12 \%$ with three patients achieving partial response; there were no complete responses. Median duration of response was 4 months; treatment-related adverse events occurred in $85 \%$ of patients, resulting in discontinuation in two patients and death in one for interstitial lung disease [85].

Similarly, nivolumab in a multicenter phase II trial reached a response rate of $8.7 \%$ and a median PFS of 4.9 months [86].

Combination of nivolumab and ipilimumab was not able to increase the response of ACC to immunotherapy, as only two patients out of 32 achieved a partial response within a phase II trial [87].

Recently, a phase 2 prospective clinical trial showed that combination of immunotherapy with hypofractionated radiation therapy for progressive, metastatic ACC didn't increase response rate, overall survival and progression free survival compared with immunotherapy alone [88].

Ongoing trials with chemotherapy, targeted agents, immunotherapy and other agents or with combinations are presented in Table 2.

\section{Conclusion}

Due to its rarity and indolent behavior, there are not consensus guidelines for the treatment of RM ACC. Thanks to biomolecular and translational research, new targets for therapy have been identified. $M Y B, N O T C H 1$ and epigenetic pathways are the most appealing targets on which research is focusing, while not impressive results have been till now obtained with drugs acting on other pathways (e.g. EGFR, FGR, CKIT and HDAC). The role of immunotherapy is under investigation, even if preliminary results confirmed the low immunogenicity of this disease.

Multicenter cooperation to study genomic pathways involved in ACC and to carry out clinical trials is essential in such a rare cancer.

\section{Declaration of Competing Interest}

Paolo Bossi: Advisory board or conference honoraria: Merck, Sanofi, Merck Sharp \& Dohme, Sun Pharma, Angelini, AstraZeneca, Bristol- 
Myers Squibb, Helsinn, GSK.

\section{Acknowledgements}

Thanks to Aashni Shah and Luca Giacomelli (Polistudium, Italy) for their assistance. This support has been paid with internal funds.

\section{Funding}

This research did not receive any specific grant from funding agencies in the public, commercial, or not-for-profit sectors.

\section{References}

[1] Coca-Pelaz A, Rodrigo JP, Bradley PJ, Vander Poorten V, Triantafyllou A, Hunt JL, et al. Adenoid cystic carcinoma of the head and neck - An update. Oral Oncol 2015; 51(7):652-61. https://doi.org/10.1016/j.oraloncology.2015.04.005.

[2] Ellington CL, Goodman M, Kono SA, Grist W, Wadsworth T, Chen AY, et al. Adenoid cystic carcinoma of the head and neck: Incidence and survival trends based on 1973-2007 Surveillance, Epidemiology, and End Results data. Cancer 2012;118(18):4444-51. https://doi.org/10.1002/cncr.27408.

[3] Ciccolallo L, Licitra L, Cantú G, Gatta G. Survival from salivary glands adenoid cystic carcinoma in European populations. Oral Oncol 2009;45(8):669-74. https:// doi.org/10.1016/j.oraloncology.2008.10.010.

[4] van Weert S, Bloemena E, van der Waal I, de Bree R, Rietveld DHF, Kuik JD, et al Adenoid cystic carcinoma of the head and neck: A single-center analysis of 105 consecutive cases over a 30-year period. Oral Oncol 2013;49(8):824-9. https://doi. org/10.1016/j.oraloncology.2013.05.004.

[5] Atallah S, Casiraghi O, Fakhry N, Wassef M, Uro-Coste E, Espitalier F, et al. A prospective multicentre REFCOR study of 470 cases of head and neck Adenoid cystic carcinoma: epidemiology and prognostic factors. Eur J Cancer 2020 May;130 (241-249). https://doi.org/10.1016/j.ejca.2020.01.023.

[6] Terhaard CHJ, Lubsen H, Van der Tweel I, Hilgers FJM, Eijkenboom WMH, Marres HAM, et al. Salivary gland carcinoma: independent prognostic factors for locoregional control, distant metastases, and overall survival: results of the Dutch head and neck oncology cooperative group. Head Neck 2004;26(8):681-93. https://doi.org/10.1002/hed.10400.

[7] van Weert S, Reinhard R, Bloemena E, Buter J, Witte BI, Vergeer MR, et al. Differences in patterns of survival in metastatic adenoid cystic carcinoma of the head and neck: Patterns in metastatic adenoid cystic carcinoma. Head Neck 2017; 39(3):456-63. https://doi.org/10.1002/hed.24613.

[8] Xu MJ, Wu TJ, van Zante A, El-Sayed IH, Algazi AP, Ryan WR, et al. Mortality risk after clinical management of recurrent and metastatic adenoid cystic carcinoma. J Otolaryngol - Head Neck Surg 2018;47(1):28. https://doi.org/10.1186/s40463018-0273-z.

[9] Cordesmeyer R, Schliephake H, Kauffmann P, Tröltzsch M, Laskawi R, Ströbel P, et al. Clinical prognostic factors of salivary adenoid cystic carcinoma: A singlecenter analysis of 61 patients. J Cranio-Maxillofac Surg 2017;45(11):1784-7. https://doi.org/10.1016/j.jcms.2017.08.004.

[10] Gao M, Hao Y, Huang MX, Ma DQ, Luo HY, Gao Y, et al. Clinicopathological study of distant metastases of salivary adenoid cystic carcinoma. Int J Oral Maxillofac Surg 2013;42(8):923-8. https://doi.org/10.1016/j.ijom.2013.04.006.

[11] Sung M-W, Kim KH, Kim J-W, Min Y-G, Seong W-J, Roh J-L, et al. Clinicopathologic predictors and impact of distant metastasis from adenoid cystic carcinoma of the head and neck. Arch Otolaryngol Neck Surg 2003;129(11):1193. https://doi.org/10.1001/archotol.129.11.1193.

[12] Girelli L, Locati L, Galeone C, Scanagatta P, Duranti L, Licitra L, et al. Lung metastasectomy in adenoid cystic cancer: Is it worth it? Oral Oncol 2017;65:114-8. https://doi.org/10.1016/j.oraloncology.2016.10.018.

[13] Hirvonen K, Bäck L, Saarilahti K, Leivo I, Hagström J, Mäkitie AA. Pattern of recurrent disease in major salivary gland adenocystic carcinoma. Virchows Arch 2015;467(1):19-25. https://doi.org/10.1007/s00428-015-1760-5.

[14] Cavalieri S, Mariani L, Vander Poorten V, Van Breda L, Cau MC, Lo Vullo S, et al. Prognostic nomogram in patients with metastatic adenoid cystic carcinoma of the salivary glands. Eur J Cancer 2020;136:35-42. https://doi.org/10.1016/j. ejca.2020.05.013.

[15] Ju J, Li Y, Chai J, Ma C, Ni Q, Shen Z, et al. The role of perineural invasion on head and neck adenoid cystic carcinoma prognosis: a systematic review and metaanalysis. Oral Surg Oral Med Oral Pathol Oral Radiol 2016;122(6):691-701. https://doi.org/10.1016/j.0000.2016.08.008.

[16] Martins-Andrade B, dos Santos Costa SF, Sant'ana MSP, Altemani A, Vargas PA, Fregnani ER, et al. Prognostic importance of the lymphovascular invasion in head and neck adenoid cystic carcinoma: A systematic review and meta-analysis. Oral Oncol 2019;93:52-8. https://doi.org/10.1016/j.oraloncology.2019.04.014.

[17] Speight PM, Barrett AW. Prognostic factors in malignant tumours of the salivary glands. Br J Oral Maxillofac Surg 2009;47(8):587-93. https://doi.org/10.1016/j. bjoms.2009.03.017.

[18] Choi Y, Kim S-B, Yoon DH, Kim JY, Lee S, Cho K-J. Clinical characteristics and prognostic factors of adenoid cystic carcinoma of the head and neck: adenoid cystic carcinoma of the head and neck. Laryngoscope 2013;123(6):1430-8. https://doi. org/10.1002/lary.23976.
[19] Szanto PA, Luna MA, White RA. Histologic grading of adenoid cystic carcinoma of the salivary glands. 1984;54(6):8. https://doi.org/10.1002/1097-0142(19840915) 54:6<1062::AID-CNCR2820540622>3.0.CO;2-E.

[20] Spiro RH, Huvos AG, Strong EW. Adenoid cystic carcinoma of salivary origin. Am J Surg 1974;128(4):512-20. https://doi.org/10.1016/0002-9610(74)90265-7.

[21] Perzin KH, Gullane P, Clairmont AC. Adenoid cystic carcinomas arising in salivary glands: a correlation of histologic features and clinical course. Cancer. 1978;42(1): 265-282. doi:10.1002/1097-0142(197807)42:1. https://doi.org/10.1002/10970142(197807)42:1<265::AID-CNCR2820420141>3.0.CO;2-Z.

[22] Jeong IS, Roh J-L, Cho K-J, Choi S-H, Nam SY, Kim SY. Risk factors for survival and distant metastasis in 125 patients with head and neck adenoid cystic carcinoma undergoing primary surgery. J Cancer Res Clin Oncol 2020;146(5):1343-50. https://doi.org/10.1007/s00432-020-03170-5.

[23] Chae YK, Chung SY, Davis AA, Carneiro BA, Chandra S, Kaplan J, et al. Adenoid cystic carcinoma: current therapy and potential therapeutic advances based on genomic profiling. Oncotarget 2015;6(35):37117-34. https://doi.org/10.18 632/oncotarget.5076.

[24] Li Q, Huang P, Zheng C, Wang J, Ge M. Prognostic significance of p53 immunohistochemical expression in adenoid cystic carcinoma of the salivary glands: a meta-analysis. Oncotarget 2017;8(17):29458-73. https://doi.org/10.186 32/oncotarget.15297.

[25] Thierauf J, Weissinger SE, Veit JA, Affolter A, Laureano NK, Beutner D, et al. Low SOX2 expression marks a distinct subset of adenoid cystic carcinoma of the head and neck and is associated with an advanced tumor stage. Ahmad A, editor. PLoS One. 2018;13(3):e0194989. https://doi.org/10.1371/journal.pone.0194989.

[26] Bazarsad S, Kim JY, Zhang X, Kim K-Y, Lee DY, Ryu MH, et al. Ataxiatelangiectasia-mutated protein expression as a prognostic marker in adenoid cystic carcinoma of the salivary glands. Yonsei Med J 2018;59(6):717. https://doi.org/ 10.3349/ymj.2018.59.6.717.

[27] Saida K, Murase T, Ito M, Fujii K, Takino H, Masaki A, et al. Mutation analysis of the EGFR pathway genes, EGFR, RAS, PIK3CA, BRAF, and AKT1, in salivary gland adenoid cystic carcinoma. Oncotarget 2018;9(24):17043-55. https://doi.org/10. 18632/oncotarget.24818.

[28] Stenman G, Persson F, Andersson MK. Diagnostic and therapeutic implications of new molecular biomarkers in salivary gland cancers. Oral Oncol 2014;50(8): 683-90. https://doi.org/10.1016/j.oraloncology.2014.04.008.

[29] Park S, Vora M, van Zante A, Humtsoe J, Kim H-S, Yom S, et al. Clinicopathologic implications of Myb and Beta-catenin expression in adenoid cystic carcinoma. J Otolaryngol - Head Neck Surg 2020;49(1):48. https://doi.org/10.1186/s40463020-00446-1.

[30] Andreasen S, Agander TK, Bjørndal K, Erentaite D, Heegaard S, Larsen SR, et al. Genetic rearrangements, hotspot mutations, and microRNA expression in the progression of metastatic adenoid cystic carcinoma of the salivary gland. Oncotarget 2018;9(28):19675-87. https://doi.org/10.18632/oncotarget.24800.

[31] Ho AS, Ochoa A, Jayakumaran G, Zehir A, Valero Mayor C, Tepe J, et al. Genetic hallmarks of recurrent/metastatic adenoid cystic carcinoma. J Clin Invest 2019; 129(10):4276-89. https://doi.org/10.1172/JCI128227.

[32] Ferrarotto R, Mitani Y, Diao L, Guijarro I, Wang J, Zweidler-McKay P, et al. Activating NOTCH1 mutations define a distinct subgroup of patients with adenoid cystic carcinoma who have poor prognosis, propensity to bone and liver metastasis, and potential responsiveness to Notch1 inhibitors. J Clin Oncol 2017;35(3): 352-60. https://doi.org/10.1200/JCO.2016.67.5264.

[33] Frerich CA, Brayer KJ, Painter BM, Kang H, Mitani Y, El-Naggar AK, et al. Transcriptomes define distinct subgroups of salivary gland adenoid cystic carcinoma with different driver mutations and outcomes. Oncotarget 2017 Dec 23; 9(7):7341-58. https://doi.org/10.18632/oncotarget.23641.

[34] www.accrf.org.

[35] Sabra MM, Sherman EJ, Tuttle RM. Tumor volume doubling time of pulmonary metastases predicts overall survival and can guide the initiation of multikinase inhibitor therapy in patients with metastatic, follicular cell-derived thyroid carcinoma: Tumor doubling time in thyroid cancer. Cancer 2017;123(15): 2955-64. https://doi.org/10.1002/cncr.30690.

[36] Laurie SA, Ho AL, Fury MG, Sherman E, Pfister DG. Systemic therapy in the management of metastatic or locally recurrent adenoid cystic carcinoma of the salivary glands: a systematic review. Lancet Oncol 2011;12(8):815-24. https://doi. org/10.1016/S1470-2045(10)70245-X.

[37] Hanna GJ, Bae JE, Lorch JH, Schoenfeld JD, Margalit DN, Tishler RB, et al. Longterm outcomes and clinicogenomic correlates in recurrent, metastatic adenoid cystic carcinoma. Oral Oncol 2020;106:104690. https://doi.org/10.1016/j. oraloncology.2020.104690.

[38] Karatzas A, Katsanos K, Maroulis I, Kalogeropoulou C, Tzorakoleftherakis E, Karnabatidis D. Multi-modality curative treatment of salivary gland cancer liver metastases with drug-eluting bead chemoembolization, radiofrequency ablation, and surgical resection: a case report. J Med Case Reports 2011;5(1):416. https:// doi.org/10.1186/1752-1947-5-416.

[39] Chen AM, Bucci MK, Weinberg V, Garcia J, Quivey JM, Schechter NR, et al Adenoid cystic carcinoma of the head and neck treated by surgery with or without postoperative radiation therapy: Prognostic features of recurrence. Int $\mathrm{J}$ Radiat Oncol 2006;66(1):152-9. https://doi.org/10.1016/j.ijrobp.2006.04.014.

[40] Kokemueller H, Eckardt A, Brachvogel P, Hausamen J-E. Adenoid cystic carcinoma of the head and neck-a 20 years experience. Int J Oral Maxillofac Surg 2004;33(1): 25-31. https://doi.org/10.1054/ijom.2003.0448.

[41] Balamucki CJ, Amdur RJ, Werning JW, Vaysberg M, Morris CG, Kirwan JM, et al. Adenoid cystic carcinoma of the head and neck. Am J Otolaryngol 2012;33(5): 510-8. https://doi.org/10.1016/j.amjoto.2011.11.006. 
[42] Vischioni B, Dhanireddy B, Severo C, Bonora M, Ronchi S, Vitolo V, et al. Reirradiation of salivary gland tumors with carbon ion radiotherapy at CNAO. Radiother Oncol 2020;145:172-7. https://doi.org/10.1016/j.radonc.2020.01.004.

[43] Palma DA, Olson R, Harrow S, Gaede S, Louie AV, Haasbeek C, et al. Stereotactic ablative radiotherapy versus standard of care palliative treatment in patients with oligometastatic cancers (SABR-COMET): a randomised, phase 2, open-label trial. Lancet 2019;393(10185):2051-8. https://doi.org/10.1016/S0140-6736(18) 32487-5.

[44] Bonomo P, Greto D, Desideri I, Loi M, Di Cataldo V, Orlandi E, et al. Clinical outcome of stereotactic body radiotherapy for lung-only oligometastatic head and neck squamous cell carcinoma: Is the deferral of systemic therapy a potential goal? Oral Oncol 2019;93:1-7. https://doi.org/10.1016/j.oraloncology.2019.04.006.

[45] Vengaloor Thomas T, Packianathan S, Bhanat E, Albert A, Abraham A, Gordy X, et al. Oligometastatic head and neck cancer: Comprehensive review. Head Neck 2020;42(8):2194-201. https://doi.org/10.1002/hed.26144.

[46] Al-Assaf H, Erler D, Karam I, Lee JW, Higgins K, Enepekides D, et al. Stereotactic body radiotherapy for medically unfit patients with cancers to the head and neck. Head Neck 2020;42(8):2050-7. https://doi.org/10.1002/hed.26138.

[47] Dillon PM, Chakraborty S, Moskaluk CA, Joshi PJ, Thomas CY. Adenoid cystic carcinoma: A review of recent advances, molecular targets, and clinical trials: Adenoid cystic carcinoma review and clinical trials. Head Neck 2016;38(4):620-7. https://doi.org/10.1002/hed.23925.

[48] Licitra L, Marchini S, Spinazzè S, et al. Cisplatin in advanced salivary gland carcinoma. A phase II study of 25 patients. Cancer. 1991;68(9):1874-1877. https:// doi.org/10.1002/1097-0142(19911101)68:9<1874::AID-CNCR2820680904>3.0. CO;2-S.

[49] Airoldi M, Pedani F, Succo G, Gabriele AM, Ragona R, Marchionatti S, et al. Phase II randomized trial comparing vinorelbine versus vinorelbine plus cisplatin in patients with recurrent salivary gland malignancies. 2002;91(3):541-7. https:// doi.org/10.1002/1097-0142(20010201)91:3<541::AID-CNCR1032>3.0.CO;2-Y.

[50] Licitra L, Cavina R, Grandi C, Di Palma S, Guzzo M, Demicheli R, et al. Cisplatin, doxorubicin and cyclophosphamide in advanced salivary gland carcinoma. Ann Oncol 1996;7(6):640-2. https://doi.org/10.1093/oxfordjournals.annonc.a010684.

[51] Wang X, Luo Y, Li M, Yan H, Sun M, Fan T. Management of salivary gland carcinomas - a review. Oncotarget 2017;8(3):3946-56. https://doi.org/10.186 32/oncotarget.13952.

[52] Locati LD, Bossi P, Perrone F, Potepan P, Crippa F, Mariani L, et al. Cetuximab in recurrent and/or metastatic salivary gland carcinomas: A phase II study. Oral Oncol 2009;45(7):574-8. https://doi.org/10.1016/j.oraloncology.2008.07.010.

[53] Pfeffer MR, Talmi Y, Catane R, Symon Z, Yosepovitch A, Levitt M. A phase II study of Imatinib for advanced adenoid cystic carcinoma of head and neck salivary glands. Oral Oncol 2007;43(1):33-6. https://doi.org/10.1016/j. oraloncology.2005.12.026.

[54] Argiris A, Ghebremichael M, Burtness B, Axelrod RS, Deconti RC, Forastiere AA. A phase 2 trial of bortezomib followed by the addition of doxorubicin at progression in patients with recurrent or metastatic adenoid cystic carcinoma of the head and neck: A trial of the eastern cooperative oncology group (E1303). Cancer 2011;117(15):3374-82. https://doi.org/10.1002/cncr.25852.

[55] Hoover AC, Milhem MM, Anderson CM, Sun W, Smith BJ, Hoffman HT, et al. Efficacy of nelfinavir as monotherapy in refractory adenoid cystic carcinoma: Results of a phase II clinical trial: Nelfinavir monotherapy in refractory adenoid cystic carcinoma. Head Neck 2015;37(5):722-6. https://doi.org/10.1002/ hed.23664.

[56] Keam B, Kim S-B, Shin SH, Cho BC, Lee K-W, Kim MK, et al. Phase 2 study of dovitinib in patients with metastatic or unresectable adenoid cystic carcinoma: Dovitinib in adenoid cystic carcinoma. Cancer 2015;121(15):2612-7. https://doi. org/10.1002/cncr.29401.

[57] Wong SJ, Karrison T, Hayes DN, Kies MS, Cullen KJ, Tanvetyanon T, et al. Phase II trial of dasatinib for recurrent or metastatic c-KIT expressing adenoid cystic carcinoma and for nonadenoid cystic malignant salivary tumors. Ann Oncol 2016; 27(2):318-23. https://doi.org/10.1093/annonc/mdv537.

[58] Ghosal N, Mais K, Shenjere P, Julyan P, Hastings D, Ward T, et al. Phase II study of cisplatin and imatinib in advanced salivary adenoid cystic carcinoma. Br J Oral Maxillofac Surg 2011;49(7):510-5. https://doi.org/10.1016/j.bjoms.2010.09.013.

[59] Lim JJ, Kang S, Lee MR, Pai HK, Yoon HJ, Lee JI, et al. Expression of vascular endothelial growth factor in salivary gland carcinomas and its relation to p53, Ki67 and prognosis. J Oral Pathol Med 2003;32(9):552-61. https://doi.org/10.1034/ j.1600-0714.2003.00073.x-i1.

[60] Siekmann AF, Lawson ND. Notch signalling and the regulation of angiogenesis. Cell Adhes Migr 2007;1(2):104-5. https://doi.org/10.4161/cam.1.2.4488.

[61] Locati LD, Perrone F, Cortelazzi B, Bergamini C, Bossi P, Civelli E, et al. A phase II study of sorafenib in recurrent and/or metastatic salivary gland carcinomas: Translational analyses and clinical impact. Eur J Cancer 2016;69:158-65. https:// doi.org/10.1016/j.ejca.2016.09.022.

[62] Thomson DJ, Silva P, Denton K, Bonington S, Mak SK, Swindell R, et al. Phase II trial of sorafenib in advanced salivary adenoid cystic carcinoma of the head and neck: Phase II trial of sorafenib in advanced adenoid cystic carcinoma. Head Neck 2015;37(2):182-7. https://doi.org/10.1002/hed.23577.

[63] Locati LD, Cavalieri S, Bergamini C, Resteghini C, Alfieri S, Calareso G, et al. Phase II trial with axitinib in recurrent and/or metastatic salivary gland cancers of the upper aerodigestive tract. Head Neck 2019;41(10):3670-6. https://doi.org/ 10.1002/hed.25891.

[64] Keam B, Kang EJ, Ahn M-J, Ock C-Y, Lee KW, Kwon JH, et al. Randomized phase II study of axitinib versus observation in patients with recurred or metastatic adenoid cystic carcinoma. J Clin Oncol. 2020;38(15_suppl):6503-6503.
[65] Tchekmedyian V, Sherman EJ, Dunn L, Tran C, Baxi S, Katabi N, et al. Phase II Study of Lenvatinib in Patients With Progressive, Recurrent or Metastatic Adenoid Cystic Carcinoma. J Clin Oncol. 2019 Jun 20;37(18):1529-37. https://doi.org/ 10.1200/JCO.18.01859.

[66] Locati LD, Galbiati D, Calareso G, Alfieri S, Singer S, Cavalieri S, et al. Patients with adenoid cystic carcinomas of the salivary glands treated with lenvatinib: Activity and quality of life. Cancer 2020 May;126(9):1888-94. https://doi.org/10.1002/ cncr.32754.

[67] van Boxtel W, Uijen M, Driessen C, Pegge S, Willems SM, Jonker M, et al. A phase II study on the efficacy and toxicity of cabozantinib in recurrent/metastatic salivary gland cancer patients. J Clin Oncol. 2020;38(15_suppl):6529-6529. https://doi. org/10.1200/JCO.2020.38.15 suppl.6529.

[68] Frierson HF, Moskaluk CA. Mutation signature of adenoid cystic carcinoma: evidence for transcriptional and epigenetic reprogramming. J Clin Invest 2013;123 (7):2783-5. https://doi.org/10.1172/JCI69070.

[69] Goncalves PH, Heilbrun LK, Barrett MT, Kummar S, Hansen AR, Siu LL, et al. A phase 2 study of vorinostat in locally advanced, recurrent, or metastatic adenoid cystic carcinoma. Oncotarget 2017;8(20):32918-29. https://doi.org/10.186 32/oncotarget.16464.

[70] Almeida LO, Guimarães DM, Martins MD, Martins MAT, Warner KA, Nör JE, et al. Unlocking the chromatin of adenoid cystic carcinomas using HDAC inhibitors sensitize cancer stem cells to cisplatin and induces tumor senescence. Stem Cell Res 2017;21:94-105. https://doi.org/10.1016/j.scr.2017.04.003.

[71] Rodriguez CP, Wu Q (Vicky), Voutsinas J, Fromm JR, Jiang X, Pillarisetty VG, et al. A phase II trial of pembrolizumab and vorinostat in recurrent metastatic head and neck squamous cell carcinomas and salivary gland cancer. Clin Cancer Res. 2020; 26(4):837-45. https://doi.org/10.1158/1078-0432.CCR-19-2214.

[72] Ho AL, Dunn L, Sherman EJ, Fury MG, Baxi SS, Chandramohan R, et al. A phase II study of axitinib (AG-013736) in patients with incurable adenoid cystic carcinoma. Ann Oncol 2016;27(10):1902-8. https://doi.org/10.1093/annonc/mdw287.

[73] Andersson, Åman, Stenman. IGF2/IGF1R signaling as a therapeutic target in MYBpositive adenoid cystic carcinomas and other fusion gene-driven tumors. Cells 2019;8(8):913. https://doi.org/10.3390/cells8080913.

[74] Thierauf J, Ramamurthy N, Jo VY, Robinson H, Frazier RP, Gonzalez J, et al. Clinically integrated molecular diagnostics in adenoid cystic carcinoma. Oncologist 2019;24(10):1356-67. https://doi.org/10.1634/theoncologist.2018-0515.

[75] Todorovic E, Dickson BC, Weinreb I. Salivary gland cancer in the era of routine next-generation sequencing. Head Neck Pathol 2020;14(2):311-20. https://doi. org/10.1007/s12105-020-01140-4.

[76] Even C, Lassen U, Merchan J, Le Tourneau C, Soria J-C, Ferte C, et al. Safety and clinical activity of the Notch inhibitor, crenigacestat (LY3039478), in an open-label phase I trial expansion cohort of advanced or metastatic adenoid cystic carcinoma. Invest New Drugs 2020;38(2):402-9. https://doi.org/10.1007/s10637-019-00739$\mathrm{x}$.

[77] Ferrarotto R, Eckhardt G, Patnaik A, LoRusso P, Faoro L, Heymach JV, et al. A phase I dose-escalation and dose-expansion study of brontictuzumab in subjects with selected solid tumors. Ann Oncol 2018;29(7):1561-8. https://doi.org/ 10.1093/annonc/mdy171.

[78] Ferrarotto R, Wirth LJ, Muzaffar J, Rodriguez CP, Xia B, Perez CA, et al. ACCURACY a phase II trial of AL101, a selective gamma secretase inhibitor, in subjects with recurrent/metastatic (R/M) adenoid cystic carcinoma (ACC) harboring Notch activating mutations (Notchmut). Ann Oncol. 2020;31 (suppl_4): S599-628. https://doi.org/10.1016/j.annonc.2020.08.1034.

[79] Gralewska P, Gajek A, Marczak A, Mikuła M, Ostrowski J, Śliwińska A, et al. PARP Inhibition Increases the Reliance on ATR/CHK1 Checkpoint Signaling Leading to Synthetic Lethality-An Alternative Treatment Strategy for Epithelial Ovarian Cancer Cells Independent from HR Effectiveness. Int J Mol Sci 2020 Dec 19;21(24): 9715. https://doi.org/10.3390/ijms21249715.

[80] Andersson MK, Mangiapane G, Nevado PT, Tsakaneli A, Carlsson T, Corda G, et al. ATR is a MYB regulated gene and potential therapeutic target in adenoid cystic carcinoma. Oncogenesis 2020 Jan 30;9(1):5. https://doi.org/10.1038/s41389020-0194-3.

[81] Xu L, Li L, Zhang J, Cai W, Zhao S, Liu S. Accumulated cytotoxicity of CDK inhibitor dinaciclib with first-line chemotherapy drugs in salivary adenoid cystic carcinoma cells. Odontology 2020;108(2):300-11. https://doi.org/10.1007/ s10266-019-00451-5.

[82] Mosconi C, de Arruda JAA, de Farias ACR, Oliveira GAQ, de Paula HM, Fonseca FP, et al. Immune microenvironment and evasion mechanisms in adenoid cystic carcinomas of salivary glands. Oral Oncol 2019;88:95-101. https://doi.org/ 10.1016/j.oraloncology.2018.11.028.

[83] Ross JS, Gay LM, Wang K, Vergilio JA, Suh J, Ramkissoon S, et al. Comprehensive genomic profiles of metastatic and relapsed salivary gland carcinomas are associated with tumor type and reveal new routes to targeted therapies. Ann Oncol 2017;28(10):2539-46. https://doi.org/10.1093/annonc/mdx399.

[84] Witte HM, Gebauer N, Lappöhn D, Umathum VG, Riecke A, Arndt A, et al. Prognostic impact of PD-L1 expression in malignant salivary gland tumors as assessed by established scoring criteria: Tumor Proportion Score (TPS), Combined Positivity Score (CPS), and immune cell (IC) infiltrate. Cancers 2020;12(4):873. https://doi.org/10.3390/cancers12040873.

[85] Cohen RB, Delord J-P, Doi T, Piha-Paul SA, Liu SV, Gilbert J, et al. Pembrolizumab for the treatment of advanced salivary gland carcinoma: findings of the phase $1 \mathrm{~b}$ KEYNOTE-028 study. Am J Clin Oncol 2018;41(11):1083-1088. https://doi.org/ 10.1097/COC.0000000000000429.

[86] Fayette J, Even C, Digue L, Geoffrois L, Rolland F, Cupissol D, et al. NISCAHN: A phase II, multicenter nonrandomized trial aiming at evaluating nivolumab (N) in two cohorts of patients (pts) with recurrent/metastatic (R/M) salivary gland 
carcinoma of the head and neck (SGCHN), on behalf of the Unicancer Head \& Neck Group. J Clin Oncol 2019;37(15 suppl):6083-6083. https://doi.org/10.1200/ JCO.2019.37.15_suppl.6083.

[87] Tchekmedyian V, Sherman EJ, Dunn L, Fetten JV, Michel LS, Kriplani A, et al. A phase II trial cohort of nivolumab plus ipilimumab in patients (Pts) with recurrent/ metastatic adenoid cystic carcinoma (R/M ACC). J Clin Oncol 2019;37(15_suppl): 6084-6084. https://doi.org/10.1200/JCO.2019.37.15_suppl.6084.
[88] Mahmood U, Bang A, Chen YH, Mak RH, Lorch JH, Hanna GJ, et al. A Randomized Phase 2 Study of Pembrolizumab With or Without Radiation in Patients With Recurrent or Metastatic Adenoid Cystic Carcinoma. Int J Radiat Oncol Biol Phys 2020 Aug 8;S0360-3016(20):34106-7. https://doi.org/10.1016/j.

ijrobp.2020.08.018. 